\title{
Role of Heparin Sodium Salt in the Modulation of Human Umbilical Cord-Derived Mesenchymal Stem Cell Differentiation
}

\author{
Madhumala Gopinath' ${ }^{1}$, Suman Kumar Nandy ${ }^{2}$, Ganesan Jothimani ${ }^{1}$, Sarubala Malayaperumal', Birendra Kumar \\ Mishra $^{3}$, Rosa Di Liddo4, Surajit Pathak ${ }^{1 *}$ \\ ${ }^{1}$ Department of Allied Health Sciences, Chettinad Hospital \& Research Institute (CHRI), Chettinad Academy of Research and \\ Education (CARE), Kelambakkam, Chennai-603103, India \\ ${ }^{2}$ Bioinformatics Centre (BIF), North-Eastern Hill University, Tura Campus, Meghalaya-794002, India \\ ${ }^{3}$ Department of Rural Development and Agricultural Production, North-Eastern Hill University, Tura Campus, Tura, \\ Meghalaya-794002, India \\ ${ }^{4}$ Department of Pharmaceutical and Pharmacological Sciences, University of Padova, Padova, Italy
}

Corresponding Author: Surajit Pathak, PhD, Associate Professor, Department of Allied Health Sciences, Chettinad Hospital \& Research Institute (CHRI), Chettinad Academy of Research and Education (CARE), Kelambakkam, Chennai-603103, India. Tel: +91-44-47429050, Email:surajit.pathak@gmail.com

Received July 9, 2019; Accepted October 14, 2019; Online Published December 5, 2019

\begin{abstract}
Introduction: The present study aims to investigate the role of low molecular weight compound heparin sodium salt (HSS) to control the differentiation of the human umbilical cord (UC) derived mesenchymal stem cells (MSCs) through possible interaction with WWTR1 protein. Materials and Methods: In order to carry out this study, the human UC-derived stem cells were isolated and characterized by stem cell specific markers and the effect of HSS was studied by altering the phenotypes of MSCs. An Insilco approach was employed to reveal the structural determination of the ligand, the WWTR1 protein binding site and to predict the strength of the interaction. After HSS treatment, WWTR1, Oct4, nanog, SOX9 gene expressions were studied using real-time polymerase chain reaction (PCR). Cell staining was performed using alizarin red to confirm the formation of osteocytes.

Results: Mineralization indicated by osteocytes was confirmed using alizarin red after the treatment of HSS. Post, HSS treatment, OCT4, Nanog, RUNX2, COL1A1 and WWTR1 gene expressions were positively modulated. Heparin treatment of MSCs lead to the up regulation of WWTR1 along with the down regulation of stemness markers Oct4 and Nanog expression. In silico studies also predicted the possible interaction of WWTR1 with HS. Results indicated that Amino acid residues ASP57, GLN83, GLN109, THR135, and TYR141 came up as a prominent interaction centre; ASP57, GLN83 and THR135 recorded the highest interaction energy - while ASP57 mostly participated in an electrostatic interaction.

Conclusions: To conclude, it can be stated that heparin can possibly interact with WWTR1 along with having the capability to direct cells towards osteogenic lineages.

Keywords: Heparin, Mesenchymal Stem Cells, WWTR1, Cell Proliferation, Osteocyte Differentiation

Citation: Gopinath M, Nandy SK, Jothimani G, et al. Role of heparin sodium salt in the modulation of human umbilical cord-derived mesenchymal stem cell differentiation. J Appl Biotechnol Rep. 2019;6(4):165-171. doi:10.29252/JABR.06.04.06.
\end{abstract}

\section{Introduction}

The ECM is a three-dimensional complex network of macromolecules including collagens, proteoglycans, elastin, and laminins ubiquitously present within all tissues and organs in the body. ${ }^{1}$

Heparin and heparan sulfate are notable extracellular polysaccharides which have recently been investigated for their catalytic role within the extracellular matrix of the cell. Heparin is found to be primarily produced in the specialized secretory cells namely mast cells, as well as stored in the intracellular granules of those cells. ${ }^{2}$ The heparin treatment has been proven to be non-toxic for mesenchymal stem cells (MSCs) which supports and leads them towards differentiation, dedifferentiation and chondrogenesis. ${ }^{3}$ They directly bind and trigger the activity of TGF-beta1 and other growth factors, which lead to mitogenic or differentiation effects of the cells. ${ }^{4}$ Previous studies have suggested that WWTR1, the hippo pathway regulator co activates RUNX2dependent transcription are found to direct MSCs towards osteogenic differentiation..$^{5-7}$

However, till now no studies have pointed out to the fact that heparin may be able to modulate WWTR1 expression in MSCs, which possibly leads to the differentiation of osteocytes. Commonly, heparin can repair and allows tissue

Copyright $\odot 2019$ The Author(s). This is an open-access article distributed under the terms of the Creative Commons Attribution License (http:// creativecommons.org/licenses/by/4.0), which permits unrestricted use, distribution, and reproduction in any medium, provided the original work is properly cited. 
regeneration to occur, placing a promising strategy for functional recovery. Also, WWTR1's fate is reversible if not degraded by the cytoplasm through phosphorylation, and is dephosphorylated back to the nucleus, which is a key attribute for its epithelial mesenchymal transition. ${ }^{8-10}$ Heparin addition has shown to increase WWTR1 expression and matrix stiffness. ${ }^{11,12}$

It is believed that heparin regulates differentiation by directing cells towards forming osteocytes and chondrocytes when human umbilical MSCs (hUCMSCs) are induced by providing the necessary growth factors. In this study, it has been aimed to investigate the possible interaction of heparin with WWTR1 which may provide additional scope for observing the differentiation potential of MSCs.

\section{Materials and Methods}

Isolation and Characterization of Mesenchymal Stem Cells In the present study, after obtaining the patient's consent, fresh umbilical cords (UCs) were retrieved during caesarean deliveries from the Department of Obstetrics and Gynecology of Chettinad Hospital and Research Institute (Chennai, India) and were processed within the optimal period of 12 hours (proposal no.10/IHEC/3-16). The UC sample was collected in a collection medium containing $10 \mathrm{~mL}$ PBS (RM7385, Dulbecco-A, HI-MEDIA) solution with $1 \mathrm{~mL}$ fetal bovine serum (FBS) and $10 \mu \mathrm{L}$ antibiotic-antimycotic solution. After transferring samples under a sterile laminar flow cell culture hood, UCs were rinsed twice in phosphatebuffered saline (PBS) containing antibiotic-antimycotic solution (Gibco) until the cord blood was cleared, and cord vessels were removed. The cords were manually minced into pieces of 1-3 $\mathrm{mm}^{3}$ and were allowed to adhere to flasks for 30 minutes. Then, they were floated in Low Glucose Dulbecco's Modified Eagle's Medium (LG-DMEM) containing 20\% FBS, and $1 \%$ antibiotic-antimycotic solution. Cord pieces were subsequently incubated at $37^{\circ} \mathrm{C}$ in humid air with $5 \% \mathrm{CO}_{2}$. The medium was changed once in every 3 days after initial plating. Fragments of UC were left undisturbed in culture and cells that were released and were further expanded and cultured. ${ }^{13}$

\section{Immunophenotypic Characterization by FACS}

The MSCs specific marker of the isolated umbilical cord mesenchymal stem cells (UC-MSCs) was determined by flow cytometry analysis. The cells were harvested and suspended in PBS and were stained with antibodies: CD73, CD105, CD14, CD34 and CD45 (Table 1). Cells were acquired and analyzed with the BD FACS ARIA II instrument.

\section{MTT Assay for the Dose Selection}

In order to determine the effect of heparin sodium salt (HSS) on cells in culture, an assay was performed using MTT. In a 96 well plate, 2000 cells/well were seeded and incubated for 48 hours and 72 hours. The control cell did not receive any HSS. After the MTT treatment, the formed intracellular formazan crystals were solubilized with DMSO and the absorbance of the solution was measured at $530 \mathrm{~nm}$ using an ELISA reader. The percentage of cell viability was calculated by using the formula; Percentages of Cell Viability $=(O D$ of drug treated sample/ OD of control sample) $\times 100$.

Treatment of Mesenchymal Stem Cells With Heparin Sodium Salt

An HSS stock solution of $1 \mathrm{uM}$ was prepared with serum freeDulbecco's modified Eagle medium. The cells were exposed to various working concentration of HSS prepared from stock solution. The drug was introduced into the medium every 48 hours and characterized on day 8 .

\section{Colony Forming Unit Assay}

The plated cells were briefly fixed with chilled methanol for about 5 minutes and were stained with Giemsa and incubated at room temperature for 10-20 minutes. Colonies were visualized using inverted microscope (Leica Microsystems).

\section{Cell Differentiation}

Induction medium and cell staining techniques was utilized to confirm the formation of osteocytes, chondrocytes and adipocytes. Alizarin Red staining for osteocytes, Toluidine blue for chondrocytes and oil red staining for adipocytes was employed. (HIMEDIA: Catalogue codes: AL521-1X100ML HiAdipoXL TM Adipocyte, AL523-1X100ML HichondroXL TM, AL522-1X100ML HiOsteoXL TM Osteocyte)

\section{Protein Modeling}

The amino acid sequence of WWTR1 were obtained and subjected to the model building using modelling servers namely IntFOLD, I-TASSER, Phyre 2 and Raptor X. ${ }^{14,15}$ The globular models shaped by I-TASSER and Phyre2 were protonated at physiological $\mathrm{pH}$ and ionic strength, subjected to loop refinement, and minimized in a smart minimizer of DS 2.5 to satisfy the root mean square gradient of $0.1 \mathrm{kcal} /$ mol A. Furthermore, both the direct server output and minimized models were validated using Structural Analysis

Table 1. FACS Antibodies

\begin{tabular}{|c|c|c|c|}
\hline Antibody & Specificity & Conjugate & Catalogue Number and Details \\
\hline CD 14 & Hematopoietic cells (negative marker) & FITC-A & Mouse anti-human CD14 Mouse IgG1, к FITC BD Biosciences 555397 \\
\hline CD90 & Mesenchymal stem cell (positive marker) & FITC-A & Mouse anti-human CD90 Mouse IgG1, к FITC BD Biosciences 555595 \\
\hline CD34 & Hematopoietic cells (negative marker) & PE-A & Mouse anti-human CD34 Mouse IgG1, к PE BD Biosciences 555822 \\
\hline CD105 & Mesenchymal stem cell (positive marker) & PerCP-Cy5-5-A & 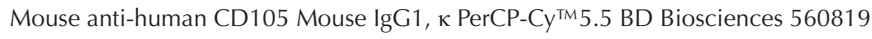 \\
\hline CD45 & Hematopoietic cells (negative marker) & APC-Cy7-A & Mouse anti-human CD45 Mouse IgG1, к FITC BD Biosciences 555482 \\
\hline
\end{tabular}


and Verification Server (SAVES) (http://services.mbi.ucla. edu/SAVES/). The I-TASSER produced the best model on the basis of stereochemistry of the models. ${ }^{16}$

\section{Protein-Ligand Docking}

The structure of Sodium Heparin [PubChem CID: 22833565] was obtained from PubChem ${ }^{17}$ and the ligand binding site of WWTR1 was predicted using $\mathrm{COACH}^{18}$ 3D Ligandsite, CASTp, DoGSiteScorer, FTSite and Sitehound servers. All possible conformers of the ligand were generated and subjected to molecular docking by Genetic Optimization for Ligand Docking (GOLD 5.2) software package on the basis of the detected binding site residues (GLU131 THR135 GLN139 TYR141 LEU143 HIS145 ILE149 TRP152) using ChemPLP and ChemScore scoring function. The best complex was chosen on the basis of ChemPLP Fitness and was minimized in smart minimizer. Also, the binding free energy and interaction energies were calculated in DS 2.5.

\section{qRT-PCR}

The RNA was extracted from the cell sample using TRIzol method. The c-DNA was synthesized using superscript cDNA synthesis kit according to the manufacturer's protocol. The qRT-PCR for the genes (Table 2) was performed using Applied Biosystem 7900 PCR system. The Ct values for target genes were normalized using endogenous control beta actin. The fold difference in the heparin treated cells was calculated by $2^{\text {-ddCt }}$ with respect to the control.

\section{Statistical Analysis}

Data was presented as mean \pm SD. Student's $t$ test was used to assess differences between the samples. $P$ values $<0.05$ was considered as a significant difference.

\section{Results}

\section{Isolation}

After 10 days of isolation, UC-derived cells were found to migrate and adhered to the surface of the flasks (Figure 1a and 1b). The isolated and expanded UC-MSCs showed spindleshaped fibroblast morphology.

\section{Surface Marker Analysis of the Cells}

The flow cytometry analysis of the isolated cells displayed a positive expression pattern for CD73, CD105 and lacked

\begin{tabular}{|c|c|}
\hline Gene & Sequence \\
\hline WWTR1 & $\begin{array}{l}\text { F- 5'GTCACGCAGGACCTAGACAC } \\
\text { R- 3'CACGAGCTAGGCTTCGGATT }\end{array}$ \\
\hline RUNX2 & $\begin{array}{l}\text { F- CAACAAGACCCTGCCCGT. } \\
\text { R- TCCCATCTGGTACCTCTCCG. }\end{array}$ \\
\hline OCT-4 & $\begin{array}{l}\text { F- 5'CGACCATCTGCCGCTTTGAG } \\
\text { R- 3'CCСССТGTCССССАTTCСТA }\end{array}$ \\
\hline NANOG & $\begin{array}{l}\text { F-5'AGTCCCAAAGGCAAACAACCCACTTC } \\
\text { R-3'ATCTGCTGGAGGCTGAGGTATTTCTGTCTC }\end{array}$ \\
\hline COL1A1 & $\begin{array}{l}\text { F- GCTACCCAACTTGCCTTCATG } \\
\text { R- TGCAGTGGTAGGTGATGTTCTGA }\end{array}$ \\
\hline B-actin & $\begin{array}{l}\text { F: 5' TTCTACAATGAGCTGCGTGTG 3' } \\
\text { R: } 5^{\prime} \text { GGGGTGTTGAAGGTCTCAAA 3' }\end{array}$ \\
\hline
\end{tabular}

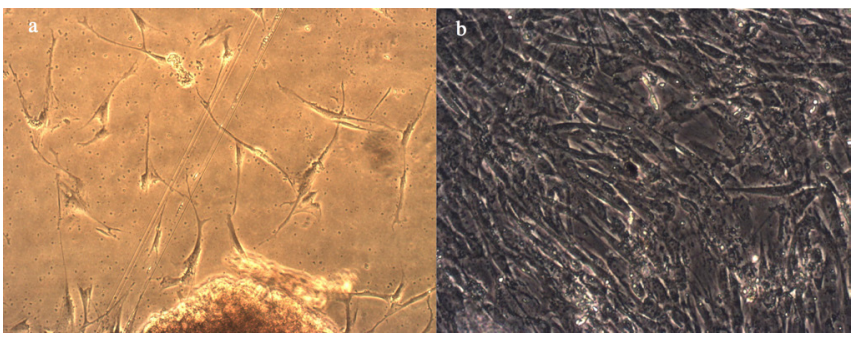

Figure 1. Stem Cells Morphology.
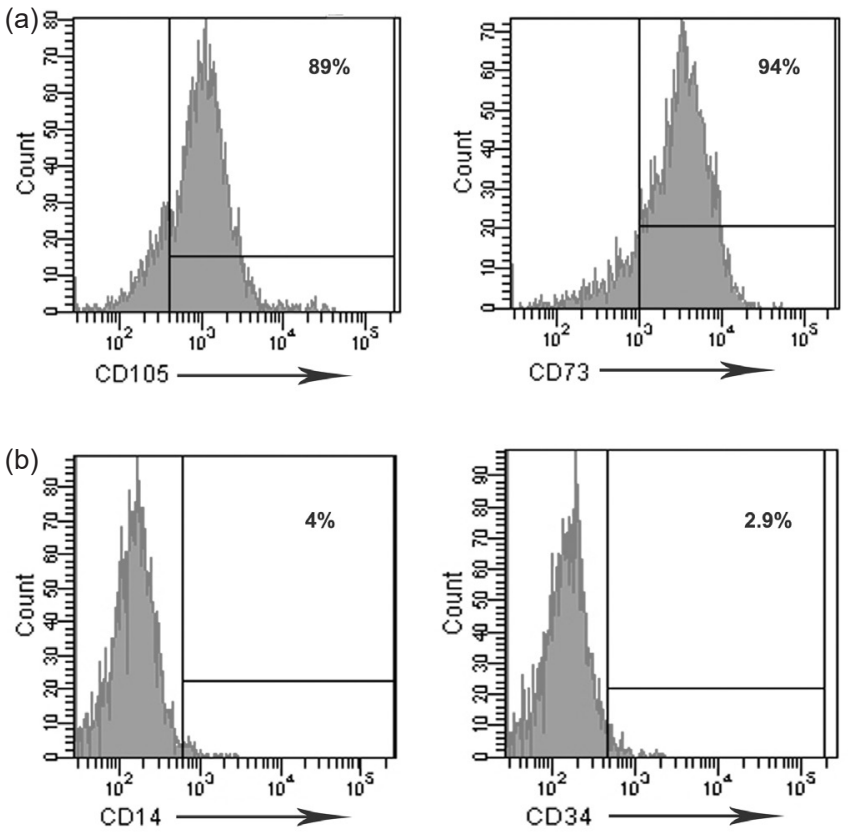

Figure 2. Characterization of MSCs. (a) CD73, CD105, (b) CD14, CD34

expression for CD14, CD34 (Figure 2) indicating that these cells were MSCs according to the minimal criteria of ISCT.

Selecting the Dose of Heparin Treatment

The $100 \mathrm{nM}$ concentration was used for this study. Human UC-derived mesenchymal cells are plastic adherent that were expanded in culture in several passages.

\section{Predictive Interaction of WWTR1- Sodium Heparin}

Electrostatic and van der Waal interactions contributed almost equally in the WWTR1-Sodium Heparin interaction profile and was characterized by numerous hydrogen bonds and $\pi$-sulfur interaction with considerable binding free energy (Tables 3, 4 and Figure 3), which corroborated the experimental findings (Figure 3 indicating experimental binding study). Amino acid residues ASP57, GLN83, GLN109, THR135, and TYR141 were found to be prominent interaction centers. However, ASP57, GLN83 and THR135 recorded the highest interaction energy. This is while ASP57 mostly participated in electrostatic interaction and the other two made both VDW and electrostatic contacts efficient (Table 4, Figure 3). The implications of heparin binding to WWTR1 and its effect in its functionality is yet to be established. Residues 


\begin{tabular}{llll}
\multicolumn{2}{l}{ Table 3. WWTR1 Interactions With Heparin Sodium } \\
\begin{tabular}{llll}
\multicolumn{2}{l}{ Hydrogen Bonds } & $\begin{array}{l}\text { I-sulphur } \\
\text { Interaction }\end{array}$ & $\begin{array}{l}\text { Binding Free Energy } \\
\text { (kcal/mol) }\end{array}$ \\
\hline Conventional & Polarized carbon & 1 & -61.76 \\
\hline 7 & 16 & 1 &
\end{tabular}
\end{tabular}

Table 4. Key Residues of Interactions

\begin{tabular}{lllll}
\hline WWTR1 & & & & \\
\hline ASP57 & HIS84 & PRO106 & THR135 & TYR141 \\
GLY81 & VAL85 & GLN108 & THR137 & \\
ALA82 & LEU94 & GLN109 & GLN139 & \\
GLN83 & LEU96 & THR133 & ARG140 & \\
\hline
\end{tabular}

with interaction energy $\geq 5 \mathrm{kcal} / \mathrm{mol}$ were reported. Residues involved in $\pi$-sulfur interaction were highlighted, amino acids exhibiting interaction energy $\geq 10 \mathrm{kcal} / \mathrm{mol}$ were labeled bold, amino acids with high electrostatic energies were noted in shaded cells, AAs with prominent van der Waals contribution were in red, AAs participating in conventional HBs were underlined and hydrogen bonds involving polarized carbon were italicized.

WWTR1 Expression after HSS Treatment

The expression of WWTR1 was significantly upregulated after heparin treatment which may be significant to the fact that the addition of heparin leading to matrix stiffness is directly proportional to WWTR1 upregulation (Figure 4).

Quantitative Real Time PCR Analysis

Heparin modulation

The present study has addressed the potential of HSS, to modulate stem cell fate of UC-derived MSCs via regulating the gene expressions of WWTR1, NANOG, OCT4, RUNX2, SOX9 and COL1A1 (Figure 4).

This study also documented the upregulation of RUNX2 gene which is the essential transcription factor for osteoblasts differentiation. Therefore, it is predicted that heparin interacts with WWTR1 thus regulating the proliferation and differentiation of MSCs (Figure 4).

Continuous Treatment for 21 Days by Heparin Directs Cells towards Osteogenic Differentiation

Mineralization indicated by osteocytes was confirmed using alizarin red (Figures $5 \mathrm{a}$ and $5 \mathrm{~b}$ ). The WWTR1 was seen to be overexpressed along with other traditional stemness markers, such as Oct4 and Nanog that enables to distinguish an undifferentiated MSC from a differentiated one, especially derived from a human UC.

\section{Discussion}

The primary goal of the present study was to explore the modulatory role of a low molecular weight compound such as HSS in the WWTR1-mediated osteocyte differentiation of MSCs. While the progressive effects of heparin and WWTR1 upon stabilization of cell fate has been explored individually, the novelty of this study was to focus on evaluating the outcomes of the synergistic effect of WWTR1 protein and

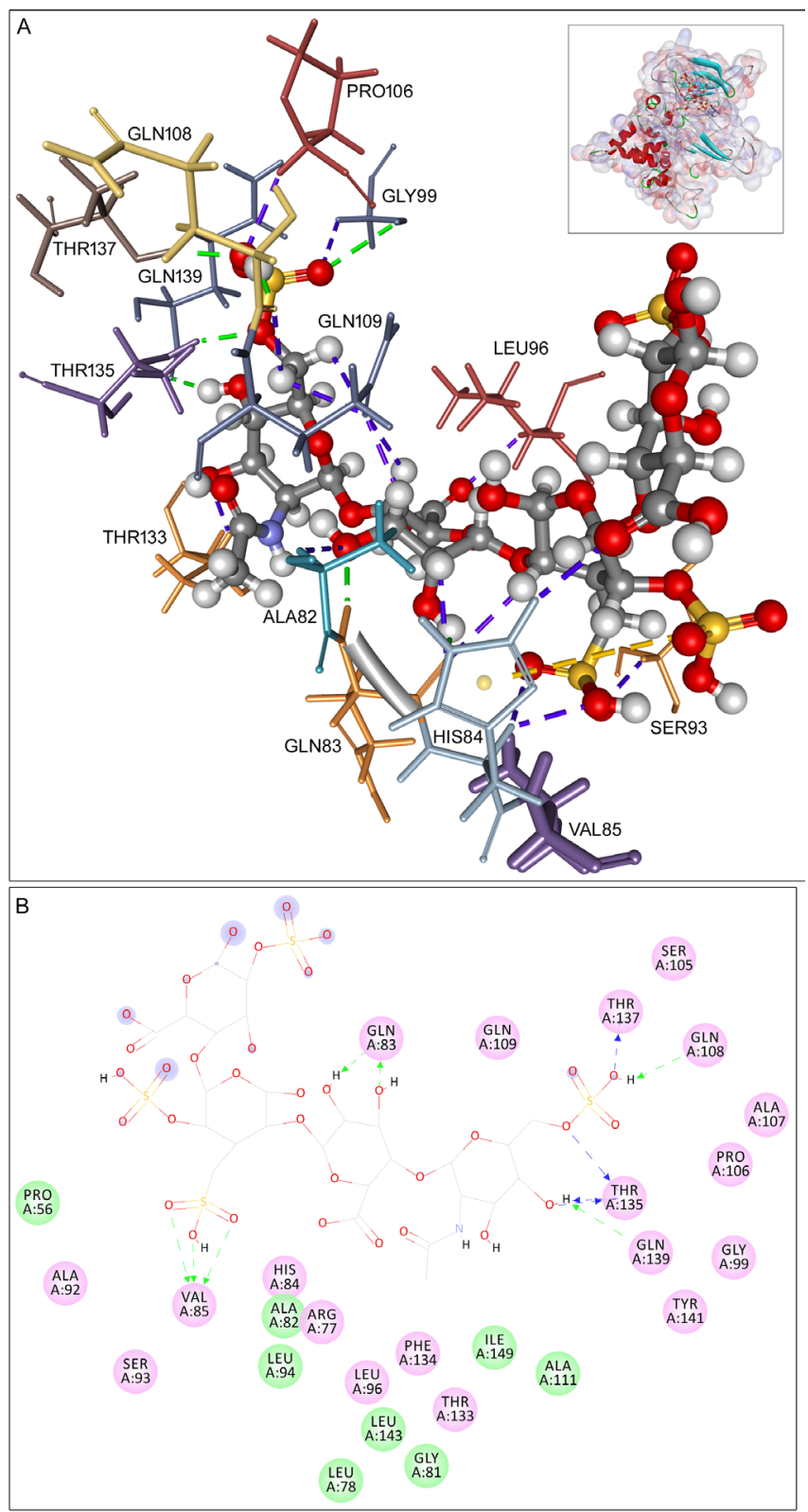

Figure 3. WWTR1-Heparin Sodium Interaction Profile
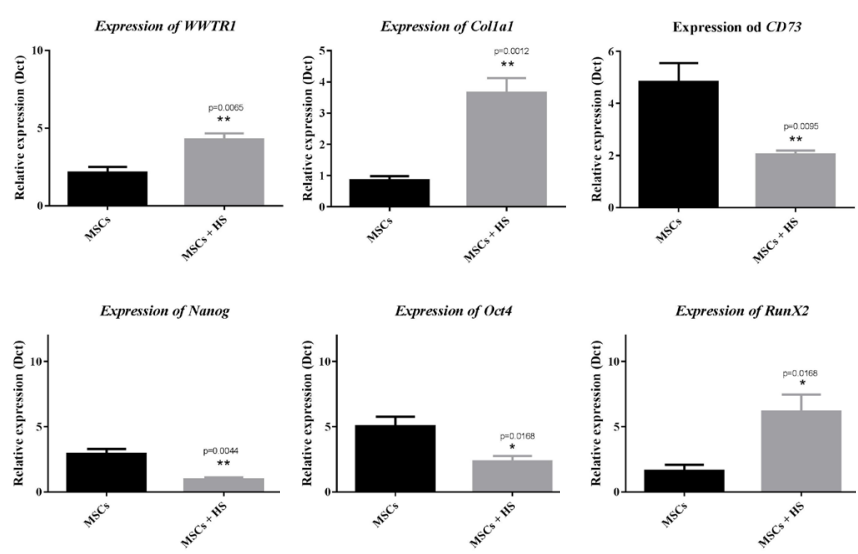

Figure 4. RT-PCR Analysis After HSS Addition. RT-PCR expression of (a) WWTR1, (b) COL1A1, (c) CD73, (d) Nanog, (e) Oct4 and (f) RUNX2 


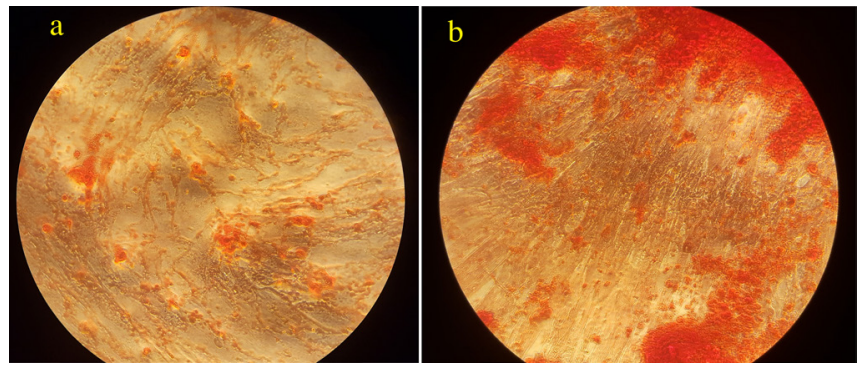

Figure 5. Osteocytes mineralization

CFU-F assay in hUCMSC

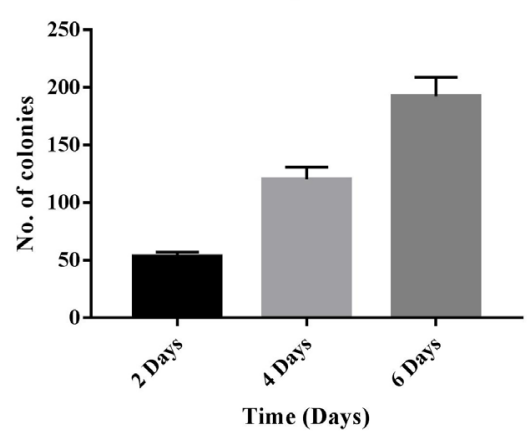

Figure 6. Cell Viability Assay.

heparin compound. Protein-ligand interaction influences the lineage commitment of stem cells as they are largely reliant on specific adhesion signals for the differentiation process. An in-depth understanding of the intricate molecular mechanics occurring in protein-ligands interactions contribute to identifying the ligand binding capacity and affinity states. The molecular functions and biological processes of target proteins are associated with their affinity for the specific ligand. It is interesting to note that osteogenic differentiation is potentially induced in UC-derived MSCs when cultured with various agents including low molecular weight compounds.

A qualitative comparative analysis was performed to view the colony forming units (CFU) (Figure 6). It is worth noting that comparative analysis of control and heparin treated cells of CFU show a higher percentage of cells that confirmed the increased proliferation of stem cells that is triggered by addition of heparin. This is in line with previous experiments where heparin has shown to increase proliferation at low doses such 200 ng. ${ }^{19,20}$

The isolated cells that showed positive for MSC markers CD90, CD73, CD105 and negative for Hematopoietic markers CD14, 34 and 45 denoted that there was no cross contamination with hematopoietic cells that were cultured. This is possibly due to the removal of the 3 vessels carrying cord blood in the human UC which is a gold standard protocol.

The WWTR1 activity increases ECM rigidity that provides the right environment for the anchoring of the osteoblasts. Thus, under the influence of heparin, signals involved in the translocation of WWTR1 into nucleus which may be deduced which can form the nucleosome complex signaling matrix proteins which have been confirmed by the phenotype of these cells. ${ }^{21}$

With the expression of PPAR $\gamma$, an adipogenic marker was downregulated in heparin treated cells. This suggests that the direction of adipogenic lineage is suppressed with the use of heparin. Chondrogenic marker SOX9 showed no change in the gene expression after heparin treatment. The RUNX2 genes are specific markers for osteogenic differentiation and RT-PCR analysis showed elevated expression of RUNX2 in heparin treated cells. This confirms that heparin may have an affinity towards forming osteocytes which is important in bone formation and heparin is an important component of bones and cartilages. The COL1A1 ECM marker was upregulated after heparin treatment which is a significant part of this study. The cell treated with heparin substantially lost its stemness marker Oct4 and Nanog expression thus the cells moves towards differentiation. It has been identified that heparin downregulates the concurrent expression of pluripotent integrators such as Nanog and Oct4 via facilitating and initiating several signaling pathways namely the fibroblast growth factor signaling. ${ }^{22}$

Surface marker analysis revealed that MSCs treated with heparin showed a variation in the surface marker expression ${ }^{2}$. Heparin has clearly shown an increase in proliferation that is in line with research on heparin where heparin has shown to increase proliferation at low doses such 200 ng. ${ }^{23}$

Finding have provided new insight into a novel mechanism of the WWTR1 involved in smad-WWTR1 axis as suggested by Park et al. The adipo-osteogenic differentiation of MSCs demonstrate a reciprocal role of WWTR1 as a positive and negative factor in osteogenesis and adipogenesis of MSCs, respectively. The WWTR1 nuclear retention leading to its active transcription regulating hippo pathway eventually leads to hard matrix formation that yields formation of osteocytes. $^{24}$ Initial findings of WWTRlexpression was in relation to RUNX2 where this protein was identified to promote osteoblast differentiation and its regulation along with repressing PPARG and adipogenesis. The findings of this study are in line with Hong et al's study. ${ }^{8}$

Further, we sought to examine the proliferation and trilineage gene expression profile of the MSCs through q-RT PCR. Stemness markers like OCT-4, NANOG, and transcriptional coactivator with PDZ-binding motif (WWTR1) were found to be downregulated in Heparin treated cells. However, the up regulation of the pre-osteoblast marker RUNX2 and major osteocyte protein COL1A1 were observed in MSCs treated with heparin indicating that HSS induces osteogenic differentiation. This result indicates that heparin treatment inhibits the proliferation of MSCs and promotes osteogenesis. Ling et al's study showed that heparin enhances osteogenesis through phosphoinositide 3-kinase/ Akt/RUNX2 pathway. ${ }^{25}$ The WWTR1 protein interacts with RUNX2 and activates transcription directing MSCs towards osteogenic differentiation.

\section{Conclusions}

This experimental study assessed the effects of heparin on the expression of WWTR1 protein in human UC derived MSCs 
and inducing differentiation. Notably, increased WWTR1 expression in MSCs significantly demonstrates WWTR1 as a cofactor for stemness. The in silico study highlights the strong binding interaction between WWTR1 and heparin. It is believed that heparin regulates differentiation by directing cells towards forming osteocytes. Despite substantial progress, further studies involving the relationship between WWTR1 and heparin via cell signaling pattern are warranted. The novelty of the undertaken study lies in the in silico approach which establishes the rationale involved in the impact of heparin-WWTR1 interaction upon the ECM and stem cell niche. The binding site, affinity and strength of the interaction results in conformational changes and validates the hypothesis. The WWTR1 protein may coactivate the expression of Runx2, a key transcription factor which drives the osteogenic differentiation. The COL1A1 is the principal gene required for providing the primary organic bone material. In this study, the expression of COL1A1 was consistently higher as the cell differentiation process began. The expression of pluripotency regulators such as nanog and oct4 is downregulated under the influence of heparin sodium salt that affects WWTR1 signalling.

\section{Authors' Contributions}

MV conducted all the experiments, analyzed the results, and wrote the drafts of the manuscript. SKN did the bioinformatics analysis. GJ, SM, RDL and BKM helped in statistical analysis and writing the MS. SP conceived the study and participated in its design, coordination and helped draft the manuscript. All authors finally reviewed the manuscript.

Conflict of Interest Disclosures

The authors declare they have no conflicts of interest.

\section{Ethics Approval}

A written informed consent was obtained from all patients and the study protocol was approved by the Institutional Review Board of Chettinad Academy of Research and Education, Chennai.

\section{Funding/Support}

This study was supported by grants from the Chettinad Academy of Research and Education, Chennai. The funders had no role in the study design, data collection and analysis, decision to publish or preparation of the manuscript.

\section{Availability of Data and Materials}

The authors declare that all relevant raw data will be freely available to any scientist wishing to use them for non-commercial purposes.

\section{Acknowledgments}

The authors are thankful to Chettinad Academy of Research and Education (CARE) for providing the research funding and infrastructure support and to DST Indo-Italian bilateral project (INT/Italy/P-5/2016 (ER) Dated: 13- 11-2017) for providing support in developing research collaboration with University of Padova to carryout experimental works.

The author would like to thank Dr. Rosy Vennila, dr. Jeswanth, Dr. Secunda, Dr. Sangeetha, Mr. Karuppasamy for their technical assistance and to Mr. Prasanna for assistance in flow cytometry analysis and to Mrs. Rajasundari, Mr. Sakthivel and Mr. Karthik for assistance in cell culture work at the Stem Cell Research Lab (CAR), Department of Surgical Gastroenterology and Liver Transplantation, Government Stanley Medical College and Hospital, India.

\section{References}

1. Järveläinen $H$, Sainio $A$, Koulu $M$, Wight $T N$, Penttinen
R. Extracellular matrix molecules: potential targets in pharmacotherapy. Pharmacol Rev. 2009;61(2):198-223. doi:10.1124/pr.109.001289.

2. da Silva EZ, Jamur MC, Oliver C. Mast cell function: a new vision of an old cell. J Histochem Cytochem. 2014;62(10):698-738. doi:10.1369/0022155414545334.

3. Kim M, Kim SE, Kang SS, Kim YH, Tae G. The use of dedifferentiated chondrocytes delivered by a heparin-based hydrogel to regenerate cartilage in partial-thickness defects. Biomaterials. 2011;32(31):7883-7896. doi:10.1016/j. biomaterials.2011.07.015.

4. McCaffrey TA, Falcone DJ, Du B. Transforming growth factor-beta 1 is a heparin-binding protein: identification of putative heparinbinding regions and isolation of heparins with varying affinity for TGF-beta 1. J Cell Physiol. 1992;152(2):430-440. doi:10.1002/ jcp.1041520226.

5. Zhu Y, Wu Y, Cheng J, et al. Pharmacological activation of TAZ enhances osteogenic differentiation and bone formation of adipose-derived stem cells. Stem Cell Res Ther. 2018;9(1):53. doi:10.1186/s13287-018-0799-z.

6. Zhao Y, Yang X. WWTR1 (WW domain containing transcription regulator 1). Atlas Genet Cytogenet Oncol Haematol. 2014;18(11):849-852. doi:10.4267/2042/54169.

7. Ehmer U, Sage J. Control of proliferation and cancer growth by the Hippo signaling pathway. Mol Cancer Res. 2016;14(2):127-140. doi:10.1158/1541-7786.mcr-15-0305.

8. Hong JH, Hwang ES, McManus MT, et al. TAZ, a transcriptional modulator of mesenchymal stem cell differentiation. Science. 2005;309(5737):1074-1078. doi:10.1126/science.1110955.

9. Oliver-De La Cruz J, Nardone G, Vrbsky J, et al. Substrate mechanics controls adipogenesis through YAP phosphorylation by dictating cell spreading. Biomaterials. 2019;205:64-80. doi:10.1016/j.biomaterials.2019.03.009.

10. Cordenonsi M, Zanconato F, Azzolin L, et al. The Hippo transducer TAZ confers cancer stem cell-related traits on breast cancer cells. Cell. 2011;147(4):759-772. doi:10.1016/j.cell.2011.09.048.

11. An EMT-scribble TAZ axis in breast cancer. In: Oren M, Aylon $Y$, eds. The Hippo Signalling Pathway and Cancer. Springer; 2013, doi:10.1007/978-1-4614-6220-0.

12. Bao M, Xie J, Katoele N, et al. Cellular volume and matrix stiffness direct stem cell behavior in a 3D microniche. ACS Appl Mater Interfaces. 2019;11(2):1754-1759. doi:10.1021/acsami.8b19396.

13. Banerjee A, Bizzaro D, Burra P, et al. Umbilical cord mesenchymal stem cells modulate dextran sulfate sodium induced acute colitis in immunodeficient mice. Stem Cell Res Ther. 2015;6:79. doi:10.1186/s13287-015-0073-6.

14. McGuffin LJ, Atkins JD, Salehe BR, Shuid AN, Roche DB. IntFOLD: an integrated server for modelling protein structures and functions from amino acid sequences. Nucleic Acids Res. 2015;43(W1):W169-173. doi:10.1093/nar/gkv236.

15. Yang J, Yan R, Roy A, Xu D, Poisson J, Zhang Y. The I-TASSER Suite: protein structure and function prediction. Nat Methods. 2015;12(1):7-8. doi:10.1038/nmeth.3213.

16. Nandy SK, Seal A. Structural dynamics investigation of human family 1 \& 2 cystatin-cathepsin L1 interaction: a comparison of binding modes. PLoS One. 2016;11(10):e0164970. doi:10.1371/ journal.pone.0164970.

17. Kim S, Thiessen PA, Bolton EE, et al. PubChem substance and compound databases. Nucleic Acids Res. 2016;44(D1):D1202-1213. doi:10.1093/nar/gkv951.

18. Yang J, Roy A, Zhang Y. Protein-ligand binding site recognition using complementary binding-specific substructure comparison and sequence profile alignment. Bioinformatics. 2013;29(20):25882595. doi:10.1093/bioinformatics/btt447.

19. Kim Yl. Comparative study for preventive effects of intra-abdominal adhesion using cyclo-oxygenase-2 enzyme (COX-2) inhibitor, low molecular weight heparin (LMWH), and synthetic barrier. Yonsei Med J. 2013;54(6):1491-1497. doi:10.3349/ymj.2013.54.6.1491. 
20. Chen YA, Lu CY, Cheng TY, Pan SH, Chen HF, Chang NS. WW domain-containing proteins YAP and TAZ in the hippo pathway as key regulators in stemness maintenance, tissue homeostasis, and tumorigenesis. Front Oncol. 2019;9:60. doi:10.3389/ fonc.2019.00060.

21. Kraushaar DC, Yamaguchi Y, Wang L. Heparan sulfate is required for embryonic stem cells to exit from self-renewal. J Biol Chem. 2010;285(8):5907-5916. doi:10.1074/jbc.M109.066837.

22. Hagmann S, Moradi B, Frank S, et al. FGF-2 addition during expansion of human bone marrow-derived stromal cells alters MSC surface marker distribution and chondrogenic differentiation potential. Cell Prolif. 2013;46(4):396-407. doi:10.1111/cpr.12046.
23. Ling L, Camilleri ET, Helledie T, et al. Effect of heparin on the biological properties and molecular signature of human mesenchymal stem cells. Gene. 2016;576(1 Pt 2):292-303. doi:10.1016/j.gene.2015.10.039.

24. Park JS, Kim M, Song NJ, et al. A reciprocal role of the Smad4-Taz axis in osteogenesis and adipogenesis of mesenchymal stem cells. Stem Cells. 2019;37(3):368-381. doi:10.1002/stem.2949.

25. Ling L, Dombrowski C, Foong KM, et al. Synergism between Wnt3a and heparin enhances osteogenesis via a phosphoinositide 3-kinase/Akt/RUNX2 pathway. J Biol Chem. 2010;285(34):2623326244. doi:10.1074/jbc.M110.122069. 